\title{
Smoothing Parametric Design of Addendum Surfaces for Sheet Metal Forming
}

\author{
Jixing $\mathrm{Li}^{1}$, Tao Ning ${ }^{1}$, Ping $\mathrm{Xi}^{1}$, Bifu Hu${ }^{1 *} \mathbb{C}$, Tian Wang ${ }^{1}$ and Jiong Yang ${ }^{2}$
}

\begin{abstract}
The manual design of addendum surfaces on common CAD platforms is very tedious which requires many trialscorrections, which will certainly affect the construction efficiency and quality of addendum surfaces, and then affect the formability and quality of the workpiece in the process of sheet forming. In this paper, an automatic procedure based on parametric design method is proposed for the rapid construction of the addendum surfaces. The kernel of the parametric method is constructing boundary curves based on the shape of surfaces of workpiece and designing guide curves based on Hermite curve interpolation. By some simple parameters, the shape of the addendum surfaces could be controlled and adjusted easily. In addition, a minimum energy optimization method is employed to further optimize the constructed addendum surface. A finite element analysis for the sheet forming process is performed to evaluate the forming quality of constructed addendum surfaces. The instance illustrates that the addendum surface constructed by the proposed method could ensure both the overall smoothing of surfaces and the final forming quality, and it has a good effect on springback after forming. This research proposes a smoothing parametric design method for addendum surfaces construction which could construct and optimize addendum surfaces rapidly.
\end{abstract}

Keywords: CAD/CAM, Parametric design, Curve optimization, Addendum surface, Sheet forming

\section{Introduction}

During the sheet forming process, stamping or incremental forming, geometric shapes exert a strong influence on the formability of the workpiece, no matter the shape of the initial sheet blank, the shape of the tools or the shape of the addendum surface $[1,2]$. An addendum surface with good quality should facilitate the global equilibrium of the sheet under the punch force, minimize the material consuming, ensure the surface smoothness, and eliminate rupture and wrinkle problems due to the strong thinning and thickening [3, 4]. Generally, addendum surfaces are manually constructed with $C A D$ software and require tedious trials-corrections, which mostly relies on the personal experience and technological standards of designers. It will be very useful to have a digital design tool for the automatic parametric design

\footnotetext{
*Correspondence: hoobye@buaa.edu.cn

${ }^{1}$ School of Mechanical Engineering and Automation, Beihang University, Beijing 100192, China

Full list of author information is available at the end of the article
}

of the addendum surfaces so that the exquisite workpiece which has good quality could be obtained with less cost.

In recent years, a bunch of literature emerged to describe some shape optimization methodology for sheet forming, such as the optimization of blank shape [5], shape optimization methodology of clinching tools [6], intelligent optimization of the drawbead geometry [7], and so on [8-10]. However, studies solely focusing on the parametric design and optimization of addendum surfaces are not so many. Among those researches, both geometric characteristics and forming effects of addendum surface's shape or parameters were studied. Li [11] studied the ISF process of geometrically complex products which had several intricate geometrical characteristics and verified that a good design of the addendum surface should be able to obtain a satisfying forming quality. Wang [12] explored some key technologies aiming at rapidly and conveniently generating addendum faces and other die surface models for FEM simulation. Using the fast Inverse Approach (IA), the Feasible Sequential Quadratic Programming (FSQP), and the Response 
Surface Method (RSM), Dong [3] and Debray [4] proposed an automatic procedure for the design and optimization of addendum surface. The profile curve technique for the parametric creation of addendum surfaces proposed by them involves some easy process parameters to improve the part's quality, and these geometric parameters are then optimized to obtain the best formability. With respect to the characteristics of multi-point forming, two methods for NURBS surface extension to design the blending surface between the surfaces of the blank holder and the workpiece were presented in Ref. [13]. Afzeri [14] studied the effect of addendum size toward the formability of the stamping process of aluminum AL6063 to prevent wrinkling and tearing. Oliveira et al. [15] performed a sensitivity study on some parameters in the blank design and indicated that the number of control points in the NURBS surface and the initial geometry have a marginal influence on the blank optimization procedure. Chi et al. $[16,17]$ presented a surface-patch method to generate addendum surface, then physics models and trans-boundary derivative vector constraints, as well as an optimization procedure based on genetic algorithm, are used for addressing smoothing problem between surface-patches.

In those study, most of the parametric design methods of addendum surfaces rely on designing composite curves formed by arcs, lines and other basic curves. To guarantee those composite curves are fully constrained, complicated geometric constraints and parameters are ineluctable. Some other methods depend on complex surface algorithms, involving overly cumbersome parameter optimization. In fact, in the perspective of CAD modeling, the modeling strategy above could only ensure the accuracy of geometric shape, but not necessarily meet the requirements of subsequent simulation and optimization, because their modeling strategy did not take the design/analysis intent [18-21], reusability $[22,23]$ and model quality [24] into account. Although this does not affect the first surface construction, it will lead to cumbersome modification iteration and optimization [24-26]. In addition, the addendum surfaces mentioned above are mainly aimed at workpiece with closed contour, which is not suitable for hatch hollow parts with semi-opening contour.

In this paper, we propose a parametric design method based on Hermite curve interpolation and minimum energy smoothing optimization for the construction of the addendum surface for a workpiece with open contour. Our method transfers the surface design problem into an optimization problem of guide curves, which reduce the number of design parameters and is easy to be implemented. The surface quality is finally evaluated in the aspects of surface smoothness and forming quality.

\section{Parametric Design Methodology of Addendum Surface}

In this paper, an aviation sheet metal part is taken as an example to show the modeling procedure and modeling strategy of the addendum surface. The given workpiece is often represented by NURBS surfaces in an IGS file, and each surface is defined by a closed contour composed of 3,4 or more NURBS curves [4].

As illustrated in Figure 1, the final part includes the desired workpiece surface, the addendum surface, the surface of the rounded die entrance and the blank holder surface. The contour of the expected workpiece connected with the blank holder surface is semi-open, which is needed to be closed. The blank holder surface is a plane parallel to XOY plane with designed boundary. The addendum surface is topologically close to $1 / 4$ spherical surface, to realize smooth transition between the expected workpiece and blank holder surface. The rounded die entrance surface could be designed finally by a simple circular bead.

In some conditions, we do not need to construct addendum surfaces deliberately for this kind of workpieces, or just perform a symmetrical operation and connection the two symmetric surfaces so that we could get two workpieces at one time. However, in some special conditions, considering the molding difficulty and cost, the addendum surface showed in Figure 1 is still necessary and has an influence on the final forming process.

Under the previous conditions, the most important part of addendum surface design is to close the contour of the workpiece, which is also the inner boundary contour of the blank holder surface, and then constructs the transition surface. We propose a concise parametric design procedure through surface frame design rather than the frequentlyused surface bridge. The proposed design procedure only requires 4 key parameters (listed in Table 1) and includes two steps: boundary curve design and guide curve design.

\subsection{Boundary Curve Design}

As showed in Figure 2, boundary curve consists of three lines and two curve fillets, which could be determined by 2 parameters, the parameter $l$ which roughly indicates the width of the addendum surface, and the parameter $r$ determines the curve fillets. Among those three lines,

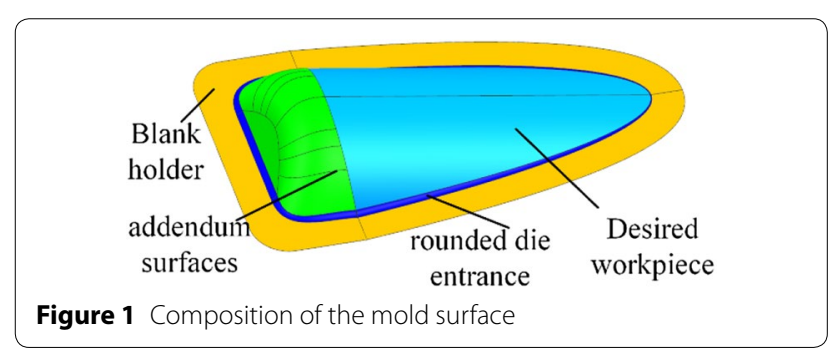


Table 1 Parameters for addendum surface design

\begin{tabular}{ll}
\hline Parameter & Description \\
\hline$r$ & The width of the addendum surface \\
$r$ & Fillet radius of the boundary curve \\
$\alpha$ & The regulatory factor of guide curves \\
$\beta$ & The regulatory factor of guide curves \\
\hline
\end{tabular}

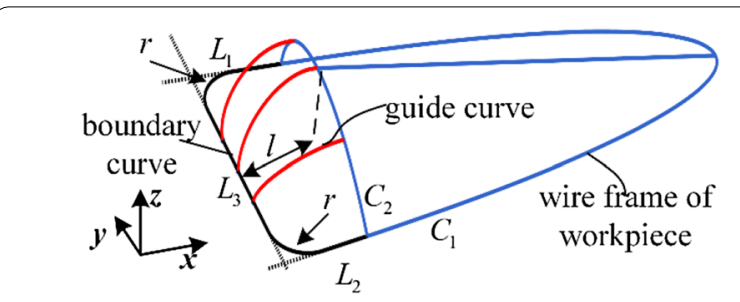

Figure 2 Parameters of addendum surface design

$L_{1}$ and $L_{2}$ are alongside the tangent directions of the endpoints of the contour curve $C_{1}$, and $L_{3}$ approximately parallel to the contour curve $C_{2}$.

In the design procedure, we should first construct a plane $B$ according to the given workpiece as the design datum, while the blank holder and other relative surface boundary are actually located in this datum plane, which means that the boundary curves must be designed in this datum plane. For the workpiece in this paper, the datum plane construction and the pre-processing of the workpiece surface are simultaneous. One of the tasks of the pre-processing is to guarantee the quality of current CAD model of the workpiece, to avoid some common mistakes such as topological fragmentation and gaps [24, 27]. Another task is to make the contour curve $C_{1}$ lie in the constructed datum plane through extending and trimming the current workpiece surfaces so that the subsequent boundary curve design could be limited to 2-D space and the design complexity would be reduced.

By identifying the endpoints of contour curve $C_{1}$ and calculating the tangent vectors at the endpoints, line $L_{1}$ and $L_{2}$ could be constructed, and they are certainly lie in the constructed datum plane $B$. As for $L_{3}$, its direction should be correlated with the contour curve $C_{2}$ to avoid overly arbitrary shapes. The $L_{3}$ could be designed based on the following steps:

Step 1: Project curve $C_{2}$ to plane $B$ to obtain a projection curve $C_{2}^{\prime}$;

Step 2: Discrete curve $C_{2}^{\prime}$ into $n$ points $p_{i}^{\prime}(i=1,2, \ldots, n)$;

Step 3: Fit points $p_{i}^{\prime}$ into a straight line $L_{3}^{\prime}$;

Step 4: Offset $L_{3}^{\prime}$ by distance $l$.

The related geometric elements involved in the construction process are shown in Figure 3.

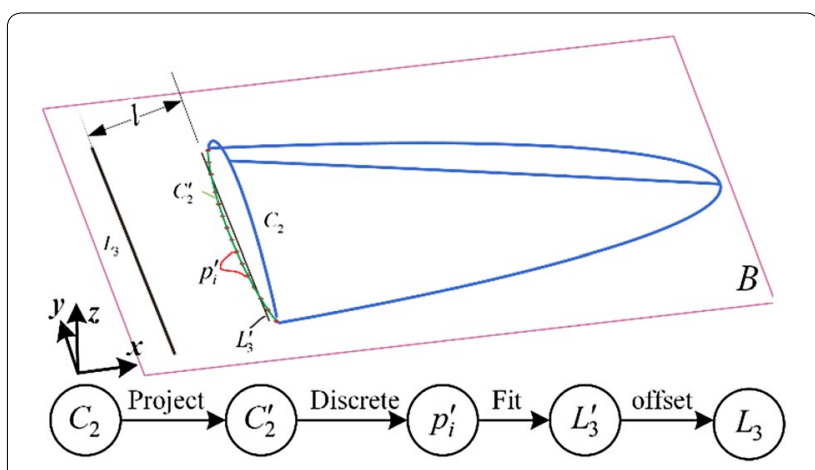

Figure 3 Construction of line $L_{3}$

Besides, it must be noted that the above boundary curve design method is only one of the feasible references, while designers must modify their design strategies according to the actual situations. For instance, the boundary curve may be required to be an over-circle curve with radius $r$. At this point, an arc which is tangent to the two endpoints of curve $C_{1}$ could be constructed with fully constraints. Then divide the arc into three parts and let the middle part correspond to the line $L_{3}$ above. Then the arc boundary is still applicable to the modeling strategy in this paper.

\subsection{Guide Curve Design}

After the boundary curve has been constructed, a series of guide curves that fulfill certain boundary conditions should be designed to connect the curve $C_{2}$ and the boundary curve, so that G1 continuity between constructed addendum surface and the given workpiece surface could be satisfied. The guide curve is designed by a Hermite curve which could be defined by an endpoint location condition and a tangent vector condition.

The Hermite curve $\boldsymbol{Q}(t)$ is a cubic spline, satisfying the following conditions:

$$
\boldsymbol{Q}\left(t_{0}\right)=\boldsymbol{P}_{0}, \boldsymbol{Q}\left(t_{1}\right)=\boldsymbol{P}_{1}, \boldsymbol{Q}^{\prime}\left(t_{0}\right)=\alpha \boldsymbol{V}_{0}, \boldsymbol{Q}^{\prime}\left(t_{1}\right)=\beta \boldsymbol{V}_{1},
$$

where $\boldsymbol{P}_{0}$ and $\boldsymbol{P}_{1}$ are given 3D endpoints, $\alpha \boldsymbol{V}_{0}$ and $\beta \boldsymbol{V}_{1}$ are given tangent vectors at $\boldsymbol{P}_{0}$ and $\boldsymbol{P}_{1}$ respectively, $\boldsymbol{V}_{0}$ and $V_{1}$ are both normalized vectors. Then $\boldsymbol{Q}(t)$ could be expressed as follows:

$$
\boldsymbol{Q}(t)=\left[F_{0}(t) F_{1}(t) G_{0}(t) G_{1}(t)\right]\left[\boldsymbol{P}_{0} \boldsymbol{P}_{1} \alpha \boldsymbol{V}_{0} \beta \boldsymbol{V}_{1}\right]^{\mathrm{T}},
$$

where 


$$
\begin{aligned}
& \left\{\begin{array}{l}
F_{0}(t)=2 s^{3}-3 s^{2}+1, \\
F_{1}(t)=-2 s^{3}+3 s^{2} \\
G_{0}(t)=s(s-1)^{2}\left(t_{1}-t_{0}\right), \\
G_{1}(t)=s^{2}(s-1)\left(t_{1}-t_{0}\right),
\end{array}\right. \\
& s=\frac{t-t_{0}}{t_{1}-t_{0}}, \quad t \in\left(t_{0}, t_{1}\right) .
\end{aligned}
$$

On the basis of Eq. (1), the guide curve could be defined by the condition $\left[\boldsymbol{P}_{0} \boldsymbol{P}_{1} \alpha \boldsymbol{V}_{0} \beta \boldsymbol{V}_{1}\right](\alpha \neq 0, \beta \neq 0)$. Due to the G1 continuity requirement, $\boldsymbol{P}_{0}$ and $\boldsymbol{P}_{1}$ must be located on a contour curve $C_{2}$ and boundary curve $L_{3}$ respectively, and at least one order continuity should be achieved at the joint of the guide curves and the workpiece surface.

Firstly, we discrete curve $C_{2}$ and $L_{3}$ into $\boldsymbol{P}_{i 0}$ and $\boldsymbol{P}_{i 1}$ $(i=1,2, \ldots, n)$ respectively according to the arc length and curvature change. Secondly, we create a datum plane $D_{i}$ through $\boldsymbol{P}_{i 0}$, while the normal vector of the plane $D_{i}$ is the tangent vector $\boldsymbol{P}_{i u}$ of the curve $C_{2}$ at $\boldsymbol{P}_{i 0}$. Thirdly, we intersect $D_{i}$ with the given workpiece surface and get intersection curves $S_{i}$. The tangent vector of $S_{i}$ at $\boldsymbol{P}_{i 0}$ is denoted as $\boldsymbol{P}_{i v}$. Finally, we denote that $\boldsymbol{V}_{i 0}=\boldsymbol{P}_{i v} /\left|\boldsymbol{P}_{i v}\right|$, $\boldsymbol{V}_{i 1}=\boldsymbol{P}_{i v} \times \boldsymbol{P}_{i u} /\left|\boldsymbol{P}_{i v} \times \boldsymbol{P}_{i u}\right|$. So far, the guide curve's boundary condition has been preliminarily defined as $\left[\boldsymbol{P}_{i 0} \boldsymbol{P}_{i 1} \alpha \boldsymbol{V}_{i 0} \beta \boldsymbol{V}_{i 1}\right]\left(\alpha=\left|\boldsymbol{P}_{i v}\right|, \beta=1\right)$, and this kind of boundary condition could make constructed addendum surface reach G1 continuous with the given workpiece. The process of constructing boundary condition and the finally G1 continuous addendum surface are illustrated in Figure 4.

On the premise that conditions $\left[\boldsymbol{P}_{0} \boldsymbol{P}_{1} \boldsymbol{V}_{0} \boldsymbol{V}_{1}\right]$ have been determined, designers could adjust the values of $\alpha$ and $\beta$ in further to optimize the shapes of the guide curves and the addendum surface, with the continuity condition unchanged. As showed in Figure 5, under different modulus conditions (different $\alpha$ or $\beta$ value), the cubic Hermite curves are obviously different. However, the cubic Hermite curves may also have loops, cusps or

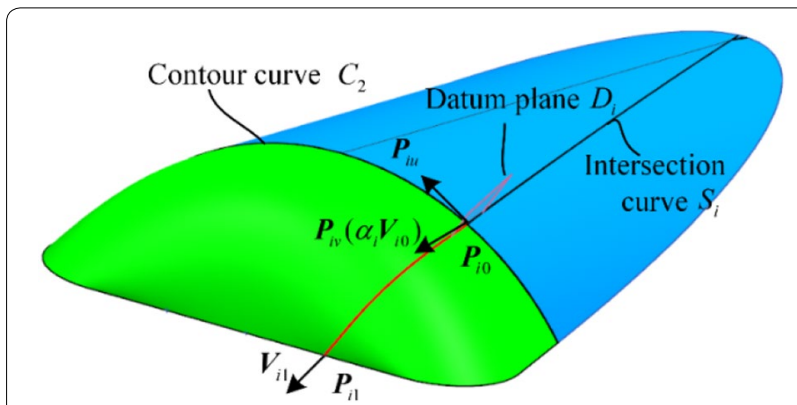

Figure 4 Determination of boundary conditions of guide curves

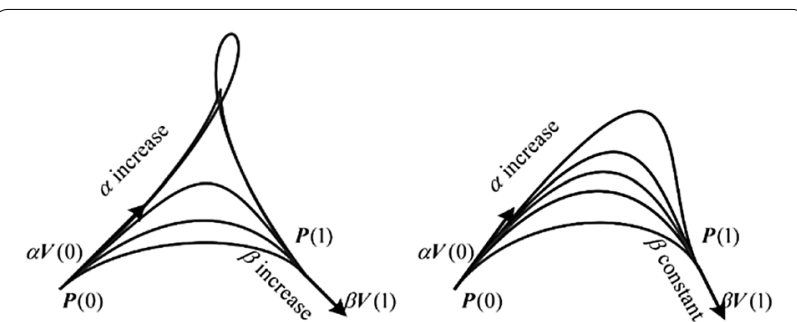

Figure 5 Influence of tangent vector modulus of curve boundary on curve shape $[28,29]$

folds in some value interval of $\alpha$ and $\beta$, which is certainly needs to be avoided.

For the problem of how the module of tangent vectors at endpoints affect the geometric characteristics of plane cubic curves, designers could refer to the conclusions given by $\mathrm{Su}$ and Liu [30]. And this solution would certainly guide designers to adjust the value of $\alpha$ and $\beta$.

Let $\alpha \boldsymbol{V}(0)=\lambda(\boldsymbol{M}-\boldsymbol{P}(0))$ and $\beta \boldsymbol{V}(1)=\mu(\boldsymbol{P}(1)-\boldsymbol{M})$, among which $M$ is the intersection point of two tangent lines, $\lambda($ or $\mu)$ denotes the relative length of the vector $\boldsymbol{M}-\boldsymbol{P}(0)$ (or $\boldsymbol{P}(1)-\boldsymbol{M})$ to the tangent vector $\alpha \boldsymbol{V}(0)$ (or $\beta \boldsymbol{V}(1))$. These symbols are illustrated in Figure 6(a), and when the value of $\lambda$ and $\mu$ are located in the region of $N 1, N 2, N 3$ and $N 4$ (see Figure 6(b)), the planar curve defined by $[\boldsymbol{P}(0) \boldsymbol{P}(1) \alpha \boldsymbol{V}(0) \beta \boldsymbol{V}(1)]$ would have no inflection point and singular point. The conversion relations of $\alpha, \beta, \lambda$ and $\mu$ are as follows:
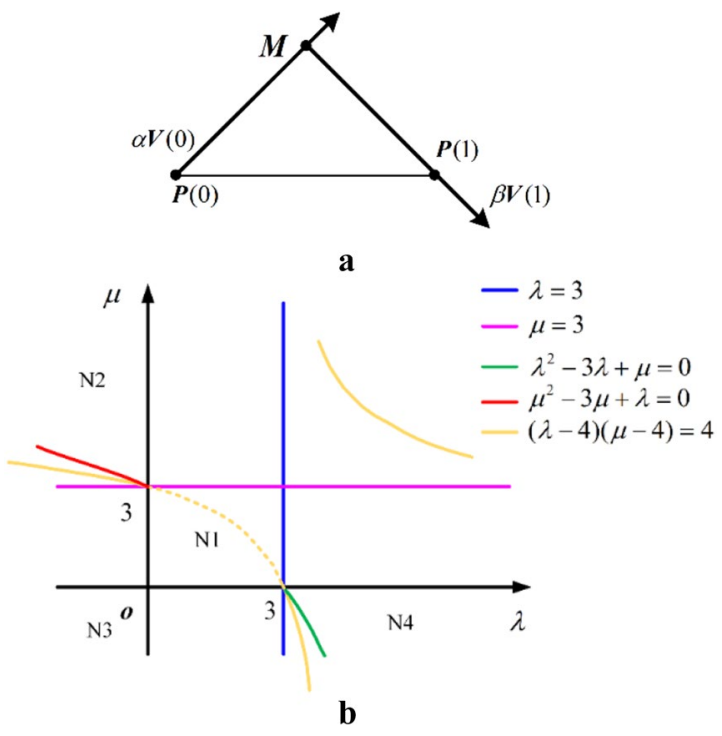

Figure 6 Interval programming of the tangential module length at the endpoint of a Hermite curve: (a) Endpoints and their tangent vectors; (b) Interval programming of curve geometric characteristics 
$\alpha=\lambda|\boldsymbol{M}-\boldsymbol{P}(0)|$,

$\beta=\mu|\boldsymbol{P}(1)-\boldsymbol{M}|$.

\section{Minimum Energy Optimization of the Guide Curves}

Although the adjustment of parameter $\alpha$ and $\beta$ could easily control the curve shape, it is still difficult for designers to obtain satisfactory guide curves that could improve the quality of the addendum surface. Thus, a minimum energy optimization method [31] is employed to optimize the guide curves.

The energy model of a cubic Hermite curve could be denoted as:

$$
E_{\text {curve }}=\int_{t_{0}}^{t_{1}}\left[\boldsymbol{Q}^{\prime \prime}(t)\right]^{2} \mathrm{~d} t .
$$

The boundary condition of cubic Hermite curve $\boldsymbol{Q}(t)$ is that $\left[\boldsymbol{P}_{0} \boldsymbol{P}_{1} \alpha \boldsymbol{V}_{0} \beta \boldsymbol{V}_{1}\right], \alpha$ and $\beta$ are uncertain. Then the problem of minimum energy optimization of the guide curves is actually that, in the curve set based on boundary condition $\left[\boldsymbol{P}_{0} \boldsymbol{P}_{1} \alpha \boldsymbol{V}_{0} \beta \boldsymbol{V}_{1}\right]$, find the $(\bar{\alpha}, \bar{\beta})$ that makes the value of $E_{\text {curve }}$ minimum. That is, the optimization variable is $\alpha$ and $\beta$, the optimization objective function is $\min f(\alpha, \beta)=\min \int_{t_{0}}^{t_{1}}\left[\boldsymbol{Q}^{\prime \prime}(t)\right]^{2} \mathrm{~d} t$.

According to Eq. (1), we can deduce that:

$$
\begin{aligned}
\boldsymbol{Q}^{\prime \prime}(t)= & \frac{\boldsymbol{P}_{0}(12 s-6)}{\left(t_{1}-t_{0}\right)^{2}}+\frac{\boldsymbol{P}_{1}(-12 s+6)}{\left(t_{1}-t_{0}\right)^{2}} \\
& +\frac{a \boldsymbol{V}_{0}(6 s-4)}{\left(t_{1}-t_{0}\right)}+\frac{b \boldsymbol{V}_{1}(6 s-2)}{\left(t_{1}-t_{0}\right)},
\end{aligned}
$$

where $s=\frac{t-t_{0}}{t_{1}-t_{0}}$. Then $f(\alpha, \beta)$ could be represented as follows:

$$
\left\{\begin{array}{l}
f(\alpha, \beta)=\frac{1}{3} A^{2}\left(t_{1}-t_{0}\right)+(A \cdot B)\left(t_{1}-t_{0}\right)+B^{2}\left(t_{1}-t_{0}\right), \\
A=\frac{12 \boldsymbol{P}_{0}}{\left(t_{1}-t_{0}\right)^{2}}-\frac{12 \boldsymbol{P}_{1}}{\left(t_{1}-t_{0}\right)^{2}}+\frac{6 \alpha V_{0}}{\left(t_{1}-t_{0}\right)}+\frac{6 \beta V_{1}}{\left(t_{1}-t_{0}\right)}, \\
B=\frac{-6 \boldsymbol{P}_{0}}{\left(t_{1}-t_{0}\right)^{2}}+\frac{6 \boldsymbol{P}_{1}}{\left(t_{1}-t_{0}\right)^{2}}-\frac{4 \alpha V_{0}}{\left(t_{1}-t_{0}\right)}-\frac{2 \beta \boldsymbol{V}_{1}}{\left(t_{1}-t_{0}\right)} .
\end{array}\right.
$$

The optimization objective function $\min f(\alpha, \beta)=$ $\min \int^{t_{1}}\left[Q^{\prime \prime}(t)\right]^{2} \mathrm{~d} t$ equates to that:

$$
\left\{\begin{array}{l}
\frac{\partial f(\alpha, \beta)}{\partial \alpha}=0, \\
\frac{\partial f(\alpha, \beta)}{\partial \beta}=0 .
\end{array}\right.
$$

Solving Eq. (4) and Eq. (6), we can get:

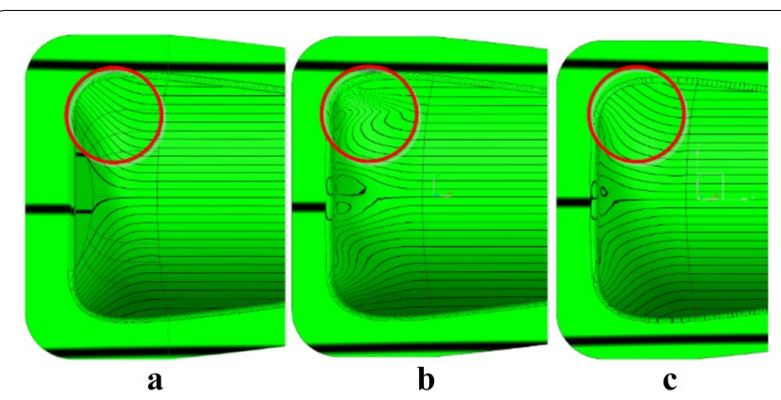

Figure 7 Smoothness of the addendum surface in different modeling method: (a) empirical scheme; (b) non-optimized scheme; (c) optimized scheme

$$
\left\{\begin{array}{l}
{\left[-\frac{6 \boldsymbol{P}_{0}}{\left(t_{1}-t_{0}\right)^{2}}+\frac{6 \boldsymbol{P}_{1}}{\left(t_{1}-t_{0}\right)^{2}}-\frac{4 \alpha \boldsymbol{V}_{0}}{\left(t_{1}-t_{0}\right)}-\frac{2 \beta \boldsymbol{V}_{1}}{\left(t_{1}-t_{0}\right)}\right] \cdot \boldsymbol{V}_{0}=0,} \\
{\left[\begin{array}{l}
\frac{12 \boldsymbol{P}_{0}}{\left(t_{1}-t_{0}\right)^{2}}-\frac{12 \boldsymbol{P}_{1}}{\left(t_{1}-t_{0}\right)^{2}}+\frac{6 \alpha \boldsymbol{V}_{0}}{\left(t_{1}-t_{0}\right)}+\frac{6 \beta \boldsymbol{V}_{1}}{\left(t_{1}-t_{0}\right)} \\
-\frac{6 \boldsymbol{P}_{0}}{\left(t_{1}-t_{0}\right)^{2}}+\frac{6 \boldsymbol{P}_{1}}{\left(t_{1}-t_{0}\right)^{2}}-\frac{4 \alpha \boldsymbol{V}_{0}}{\left(t_{1}-t_{0}\right)}-\frac{2 \beta \boldsymbol{V}_{1}}{\left(t_{1}-t_{0}\right)}
\end{array}\right] \cdot \boldsymbol{V}_{1}=0 .}
\end{array}\right.
$$

Finally, we obtain the optimum solution as follows:

$$
\left\{\begin{array}{l}
\bar{\alpha}=\frac{6\left[\left(\boldsymbol{P}_{1}-\boldsymbol{P}_{0}\right) \boldsymbol{V}_{0}\right]\left(\boldsymbol{V}_{1}^{2}\right)-3\left[\left(\boldsymbol{P}_{1}-\boldsymbol{P}_{0}\right) \boldsymbol{V}_{1}\right]\left(\boldsymbol{V}_{0} \boldsymbol{V}_{1}\right)}{\left[4 \boldsymbol{V}_{0}^{2}\left(\boldsymbol{V}_{1}^{2}\right)-\left(\boldsymbol{V}_{0} \boldsymbol{V}_{1}\right)^{2}\right]\left(t_{1}-t_{0}\right)}, \\
\bar{\beta}=\frac{3\left[\left(\boldsymbol{P}_{1}-\boldsymbol{P}_{0}\right) \boldsymbol{V}_{0}\right]\left(\boldsymbol{V}_{0} \boldsymbol{V}_{1}\right)-6\left[\left(\boldsymbol{P}_{1}-\boldsymbol{P}_{0}\right) \boldsymbol{V}_{1}\right]\left(\boldsymbol{V}_{0}^{2}\right)}{\left[\left(\boldsymbol{V}_{0} \boldsymbol{V}_{1}\right)^{2}-4 \boldsymbol{V}_{0}^{2}\left(\boldsymbol{V}_{1}^{2}\right)\right]\left(t_{1}-t_{0}\right)} .
\end{array}\right.
$$

Hence, among cubic Hermite curves defined by condition $\left[\boldsymbol{P}_{0} \boldsymbol{P}_{1} \alpha \boldsymbol{V}_{0} \beta V_{1}\right]$, the curve in condition $\left[\boldsymbol{P}_{0} \boldsymbol{P}_{1} \bar{\alpha} \boldsymbol{V}_{0} \bar{\beta} \boldsymbol{V}_{1}\right]$ has the minimum energy and the best smoothness.

Based on this optimization method, designers just need to determine the endpoints and their tangent direction $\left[\boldsymbol{P}_{0} \boldsymbol{P}_{1} \boldsymbol{V}_{0} \boldsymbol{V}_{1}\right]$, then the optimal modulus of the tangent vectors could be calculated automatically. Moreover, from Eq. (8) we can find that the computational complexity of the solution process is very small, which means that the optimization method is very fast and suitable for engineering practice.

\section{Instance Verification}

We take the workpiece showed in Figure 1 as an instance to verify the proposed parametric design method.

\subsection{Analysis of Geometric Characteristics}

As illustrated in Figure 7, three addendum surfaces are respectively constructed with the same boundary curve defined by parameter $r$ and $l$ (See Figure 2). The surface continuity and smoothness are the main evaluation criterions for surface quality. G1 continuity condition is easy to satisfy for both the proposed method and empirical manual method on the CAD platform. As for smoothness condition, the proposed method could guarantee 
its smoothness through adjusting the boundary conditions of guide curves, the values of tangent vector modulus mentioned in Section 3. As we can see in Figure 7, the addendum surface in Figure 7(b) and Figure 7(c) is designed by the proposed parametric method while the guide curves in Figure 7(c) are optimized by the minimum energy optimization method mentioned above, while the surface in Figure 7(a) is constructed by empirical design with much higher time consumption than the proposed parametric method. Represented by light reflection, the smoothness condition of the addendum surface in Figure 7(a) and Figure 7(c) is much better than in Figure 7(b), especially at the region marked with a red circle. The surface in Figure 7 (a) is slightly better than Figure 7(c) due to its complex detail design and repeated modification, but overall, it is not much different.

We further analyzed the shape and curvature distribution of the curves on the symmetrical axis of the addendum surface to evaluate the merits and demerits of the three schemes. Seen from Figure 8, we can find that the shape and curvature of the optimized guide curve and non-optimized curve change gently while the shape of the empirical curve is sharper, which will certainly lead to different material flow effect. And compared with the non-optimized guide curve, the optimized guide curve is smoother in shape and more shallow in depth, which is conducive to sheet metal forming, and the subsequent simulation also confirms it. To sum up, in the view of the surface geometric characteristics, the proposed parametric design method based on Hermite curve interpolation

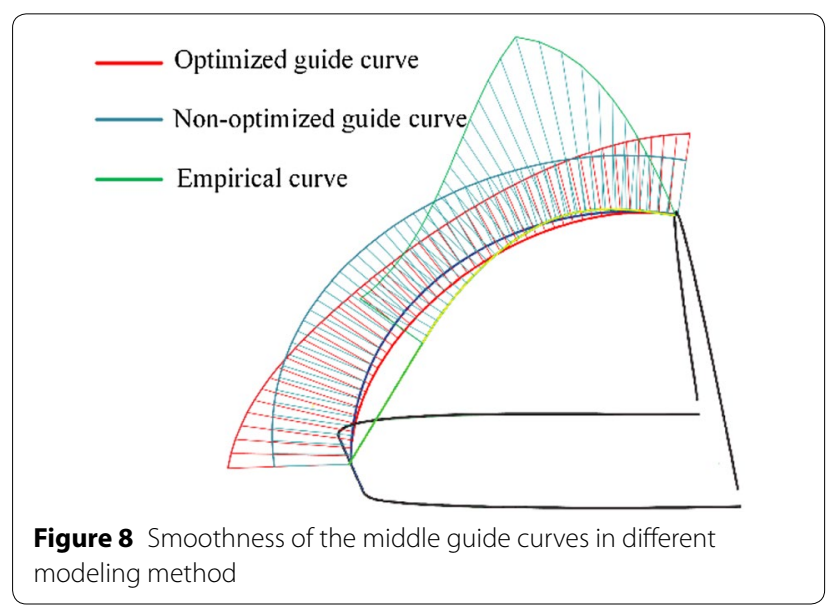

and minimum energy smoothing optimization are completely feasible for addendum surface construction.

\subsection{Finite Element Simulation of the Forming Process}

To further evaluate the forming quality of the addendum surface constructed by the proposed parametric method, the finite element analysis method is employed to simulate the forming process with the same boundary condition. Some important boundary conditions are listed in Table 2. The thickness distribution on the sheet metals of the three schemes is illustrated in Figure 9, from which we can find that:

1) The thickness distribution on the workpiece part of the three scheme is almost the same in the whole;

2) The thinnest area of the sheet metal is roughly located at the joint area of the workpiece surface and the addendum surface, and a particular round dies entrance area. Moreover, the thickness reduction of the optimized guide curve scheme is better than the other two schemes in the joint area. As for the round die entrance area, the optimized guide curve scheme is not inferior to the empirical scheme and far better than the non-optimized guide curve scheme.

To further assess the thickness reduction situation clearly, we extract the thickness distributed on a critical path, and the thickness distribution results demonstrate the superiority of the optimized guide curve scheme more intuitively. The thickness distribution along the specified path is shown in Figure 10.

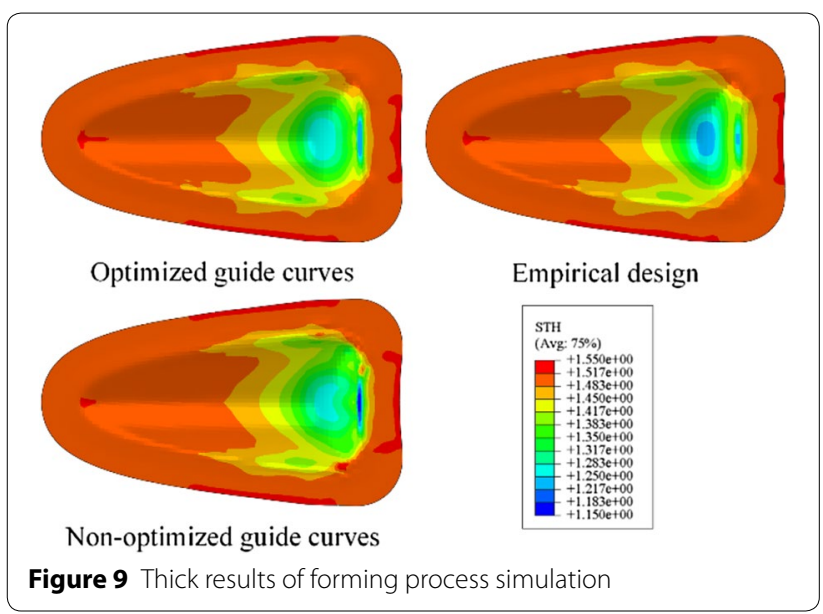

Table 2 Sheet material parameters and boundary condition

\begin{tabular}{llllll}
\hline Density $\mathbf{( k g / \mathbf { m } ^ { \mathbf { 3 } } )}$ & Poisson's ratio & $\begin{array}{l}\text { Yong's modulus } \\
\text { (MPa) }\end{array}$ & $\begin{array}{l}\text { Plastic } \\
\text { (Yield stress, plastic strain) }\end{array}$ & $\begin{array}{c}\text { Friction coefficient } \\
\text { Thickness } \\
(\mathbf{m m})\end{array}$ \\
\hline $7.8 \times 10^{3}$ & 0.3 & $210 \times 10^{3}$ & $(200,0.0)(250,0.05)(280,0.1)(300,0.2)$ & 0.4 & 1.5 \\
\hline
\end{tabular}




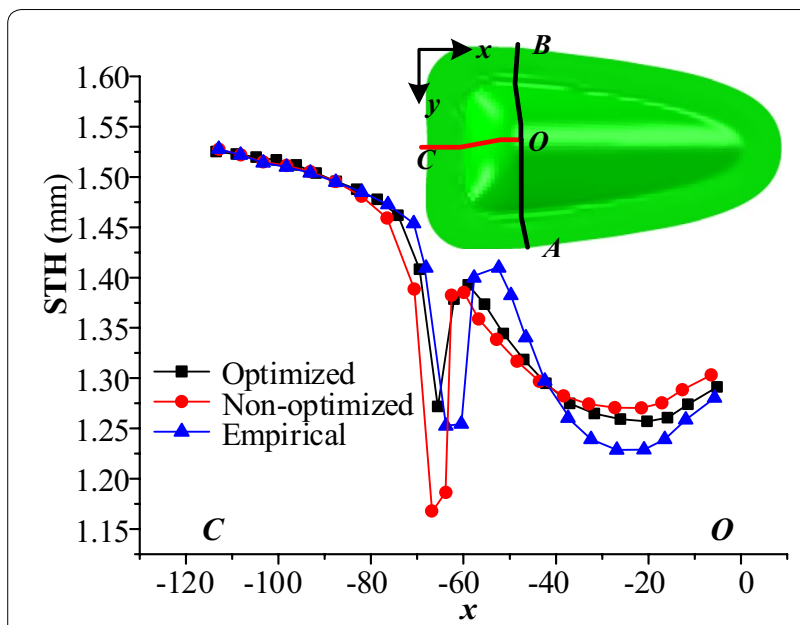

Figure 10 The thick distribution along the path CO

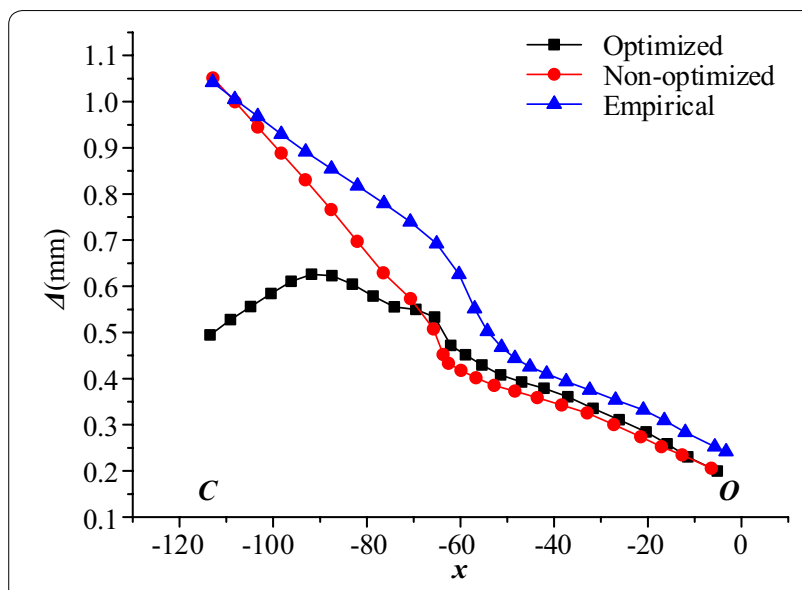

Figure 11 Springback along the path CO

Furthermore, we simulated the springback process of the three schemes. The spatial displacement $\Delta$ of each node in path $\mathrm{CO}$ is used to represent the springback of forming. Seen from Figure 11, we can conclude that the springback of the optimized scheme is significantly smaller than the other two schemes, including point $O$. It illustrates that energy-optimized addendum surface can indeed reduce the energy accumulation in the forming process, thus improve the springback, and this conclusion coincides with the original intention of the physical design of energy optimization theory.

\section{Conclusions}

(1) A concise and effective parametric design methodology of addendum surfaces could improve design quality and reduce the design cost. In this paper, a parametric design method of addendum surface based on Hermite curve interpolation and minimum energy smoothing optimization was proposed. The parametric method requires only a few design steps and control variables, therefore easy to be implemented. Both the geometric characteristics and the influence on the forming process of the constructed addendum surface were evaluated and analyzed.

(2) In the aspect of geometric characteristics, geometric continuity, and smoothness, the proposed method shows a good quality no worse than complex empirical design method. In addition to meeting basic G1 continuous requirement, the addendum surface with optimized guide curves also has a uniform shape transition, which proves the validity and reliability of the proposed parametric method.

(3) In the forming simulation, the addendum surface with optimized guide curves shows many advantages. It has better material fluidity, thus its thickness reduction on some dangerous area is superior to other schemes. Moreover, the addendum surface based on guide curves with minimum strain energy would accumulate less energy in the forming process, and it thus has a smaller springback.

\section{Limitation and Future Works}

Although the proposed parametric design method for addendum surfaces shows a good design effect. There are some more research should be performed for further perfection. Firstly, the current methodology mainly focuses on semi-open aeronautical parts, and more sheet metal parts with various functions and more complex shapes should also be verified with the method to illustrate the engineering applicability of the proposed method. Secondly, the evaluation of forming quality is mainly based on the simulation data at key areas of the parts, such as the thick distribution and springback on the critical path. However, only these assessment data are not sufficient to fully demonstrate the differences between the different schemes. Thus, in our future work, we will try to introduce new assessment tools to assist designers in evaluating design schemes. And certainly, experiments will also be performed to do the final verification and arbitration.

\section{Authors' Contributions}

$J L$ was in charge of the whole trial and wrote the whole manuscript; TN and PX were responsible for the optimization algorithm. All authors read and approved the final manuscript.

\section{Authors' Information}

Jixing Li, born in 1990, is currently a PhD candidate at School of Mechanical Engineering and Automation, Beihang University, China. His research interests include CAD/CAE integration, CAGD, and aeronautical product design. 
Tao Ning, born in 1967, is currently an associate professor at Beihang University, China. He received his PhD degree on CAD/CAM from Beihang University, China, in 1996 and has been a visiting scholar at Concordia University, Canada. His research interests include CAD/CAGD/CAM.

Ping Xi, born in 1954, is currently a professor and PhD advisor at Beihang University, China. She received her master and the PhD degree in Ecole Nationale Supérieure d'Arts et Métiers, France. Her research interests include CAD/ CAGD/CAE as well as aeronautical product design.

Bifu Hu, born in 1980, is currently an associate professor at Beihang University, China. He received his PhD degree on CAD/CAE from Beihang University, China, in 2008.

Tian Wang, born in 1992, is currently a PhD candidate at School of Mechanical Engineering and Automation, Beihang University, China. His research interests include CAD/CAE integration and blade conjugated heat transfer design.

Jiong Yang, born in 1990, is currently an associate professor at School of Mechanical Engineering, Zhengzhou University, China. His research interests include CAD, CAGD and aeronautical product design.

\section{Acknowledgements}

The authors sincerely thanks to Prof. Wei Wang and Prof. Bao Meng for their critical discussion, and Dr Anlin Long for his guide in simulation.

\section{Competing Interests}

The authors declare no competing financial interests.

\section{Funding}

Supported by National Natural Science Foundation of China (Grant No. 61471024), National Natural Science Foundation for Young Scientists of China (Grant No. 51705469).

\section{Author Details}

1 School of Mechanical Engineering and Automation, Beihang University, Beijing 100192, China. ${ }^{2}$ School of Mechanical Engineering, Zhengzhou University, Zhengzhou 450001, China.

Received: 17 July 2019 Revised: 15 October 2019 Accepted: 27 December 2019

Published online: 03 February 2020

\section{References}

[1] Y Xia, Y Wu, M A N Hendriks. Simultaneous optimization of shape and topology of free-form shells based on uniform parameterization model. Autom. Constr., 2019, 102: 148-159.

[2] Q G Le, B Raghavan, P Breitkopf. A manifold learning-based reduced order model for springback shape characterization and optimization in sheet metal forming. Computer Methods in Applied Mechanics and Engineering, 2015, 285: 621-638.

[3] M Dong, K Debray, Y Q Guo, et al. Design and optimization of addendum surfaces in sheet metal forming process. International Journal for Computational Methods in Engineering Science \& Mechanics, 2007, 8(4): 211-222.

[4] K Debray, Y M Li, Y Q Guo. Parametric design and optimization of addendum surfaces for sheet metal forming process. International Journal of Material Forming, 2013, 6(3): 315-325.

[5] S Kitayama, H Koyama, K Kawamoto,et al. Optimization of blank shape and segmented variable blank holder force trajectories in deep drawing using sequential approximate optimization. International Journal of Advanced Manufacturing Technology, 2017, 91(5-8): 1809-1821.

[6] M H Wang, G Q Xiao, Z Li, et al. Shape optimization methodology of clinching tools based on bezier curve. International Journal of Advanced Manufacturing Technology, 2018, 94(5-8): 2267-2280.

[7] Z Wang, Q C Zhang, Y Q Liu, et al. A robust and accurate geometric model for automated design of drawbeads in sheet metal forming. Computer-Aided Design, 2017, 92: 42-57.
[8] H Qin, Z J Liu, H L Zhong, et al. Two-level multiple cross-sectional shape optimization of automotive body frame with exact static and dynamic stiffness constraints. Structural and Multidisciplinary Optimization, 2018, 58(5): 2309-2323.

[9] P Kang, S K Youn. Isogeometric topology optimization of shell structures using trimmed nurbs surfaces. Finite Elements in Analysis \& Design, 2016, 120: 18-40.

[10] Y Liu, M Shimoda. Two-step shape optimization methodology for designing free-form shells. Inverse Problems in Science and Engineering, 2015, 23(1): 1-15.

[11] J Li, J Shen, B Wang. A multipass incremental sheet forming strategy of a car taillight bracket. International Journal of Advanced Manufacturing Technology, 2013, 69(9-12): 2229-2236.

[12] W Wang, G Zhao, F Shi. Die surface design cad software oriented for sheet metal stamping fem. Steel Research International, 2010, 81(9): 678-681.

[13] L Peng, X Lai, M Li. Transition surface design for blank holder in multipoint forming. International Journal of Machine Tools \& Manufacture, 2006, 46(12): 1336-1342.

[14] Afzeri, M S Shahdan, H S Qasim. Effect of addendum parameters to the formability of aluminum al 6303. Advanced Materials Research, 2011, 264265: 206-211.

[15] M C Oliveira, R Padmanabhan, A J Baptista, et al. Sensitivity study on some parameters in blank design. Materials \& Design, 2009, 30(4):1223-1230.

[16] D Chi, R Liu, P Hu, et al. Smoothing parametric method to design addendum surface. International Conference on Intelligent Computation Technology and Automation, 2008: 1140-1144.

[17] K Hu, C Di. Addendum surface design based on the parametric method. Communications in Computer \& Information Science, 2011, 144: 279-284.

[18] Y Cheng, F Z He, X Lv,et al. An information model for presenting multi-user design intents for feature-based 3D collaborative designing. New York: Assoc. Computing Machinery, 2017.

[19] Z Pan, X Wang, R Teng, et al. Computer-aided design-while-engineering technology in top-down modeling of mechanical product. Computers in Industry, 2016, 75: 151-161.

[20] J D Camba, M Contero. Assessing the impact of geometric design intent annotations on parametric model alteration activities. Computers in Industry, 2015, 71: 35-45.

[21] D C Nolan, C M Tierney, C G Armstrong, et al. Defining Simulation Intent. Computer-Aided Design, 2015, 59: 50-63.

[22] J D Camba, M Contero, P Company. Parametric cad modeling: An analysis of strategies for design reusability. Computer-Aided Design, 2016, 74: 18-31.

[23] C Zhang, G Zhou. A view-based 3D CAD model reuse framework enabling product lifecycle reuse. Advances in Engineering Software, 2019, 127: 82-89.

[24] C Gonzalez-Lluch, P Company, M Contero, et al. A survey on 3D CAD model quality assurance and testing tools. CAD Computer Aided Design 2017, 83: 64-79.

[25] Z R Cheng, Y S Ma. Explicit function-based design modelling methodology with features. Journal of Engineering Design, 2017, 28(3): 205-231.

[26] Z R Cheng, Y S Ma. A functional feature modeling method. Adv. Eng. Inform., 2017, 33: 1-15.

[27] K Rajab, L A Piegl, V Smarodzinava. CAD model repair using knowledgeguided nurbs. Engineering with Computers, 2013, 29(4): 477-486.

[28] G Farin, J Hoschek, M S Kim. Hand book of computer aided geometric design. Elsevier, 2002

[29] F Shi. Computer aided geometric design and non-uniform rational B-spline: Second edition. Beijing: Higher Education Press, 2013. (in Chinese)

[30] B Q Su, D Y Liu. Computational geometry. Shanghai: ShangHai Scientific and Technical Publishers, 1981. (in Chinese)

[31] J H Yong, F Cheng. Geometric hermite curves with minimum strain energy. Computer Aided Geometric Design, 2004, 21(3): 281-301. 